\title{
Quantitative determination of activated coagulation factor XI as an impurity in therapeutic immunoglobulins from Chinese blood fractionation companies
}

\author{
L. Ma, P. Sun, F.Z. Lin, G. Diao and C.Q. Li \\ Institute of Blood Transfusion, Chinese Academy of Medical Sciences, \\ Chengdu, Sichuan, China \\ Corresponding author: C.Q. Li \\ E-mail: lichangqing268@163.com
}

Genet. Mol. Res. 12 (3): 2556-2561 (2013)

Received May 2, 2012

Accepted September 2, 2012

Published January 4, 2013

DOI http://dx.doi.org/10.4238/2013.January.4.17

\begin{abstract}
Residual activated coagulation factor XI (FXIa) has been suggested to play an important role in thromboembolic events associated with the use of intravenous immunoglobulin (IVIG) lots. This study investigated the predominant plasma proteases in 42 IVIG lots from 4 Chinese manufacturers. In one-stage clotting assays, the procoagulant activities of factors II, VII, IX, X, XI, and XII were quantified. Non-activated partial thromboplastin time and a modified thrombin generation test served as global and FXIaspecific clotting assays, respectively. We found that coagulation factor clotting activities of the 42 IVIG lots were below the detection limit of the assays, except for the products of manufacturer B (lots of 2010), in which 0.030 to $0.032 \mathrm{IU} / \mathrm{mL}$ FXI:C were detected. The peak time of thrombin using a thrombin generation test was greater than $35 \mathrm{~min}$, the relevant amount of FXIa was below 0.37 $\mathrm{nM}$, and non-activated partial thromboplastin time was greater than 200 s. Consequently, the 42 IVIG lots showed non-significant
\end{abstract}


procoagulant potential. Further study is required to determine whether a program for FXIa determination in IVIG products should be launched in China.

Key words: Activated coagulation factor XI; Thromboembolic events; Intravenous immunoglobulin; Thrombin generation test

\section{INTRODUCTION}

The intravenous immunoglobulin (IVIG), a human plasma-derived medicinal product, is mainly used to treat primary immunodeficiency syndromes and a variety of autoimmune, infectious, transplantation-related, and chronic diseases (Brannagan et al., 1996). A small number of thromboembolic events (TEEs) occurring after the administration of IVIG preparations and infusions have been reported (Dalakas, 1994; Rosenbaum, 1997; Hefer and Jaloudi, 2004; Vo et al., 2006). These TEEs have been attributed to an increased residual content of activated procoagulant components - mainly activated coagulation factor XI (FXIa) - in IVIG (Bolton-Maggs et al., 1995; Wolberg et al., 2000). A number of tests have long been used to assess the procoagulant activity of blood derivatives, particularly those with a significant risk of thrombogenicity. However, such tests might be inapplicable for the characterization of the IVIG (Cochrane and Griffin, 1979; The European Pharmacopoeia, 2009). Recent modifications to the established thrombin generation test (TGT) have been developed to enhance the sensitivity of these tests in IVIG - for example, to detect minor quantities of potential activators such as FXIa.

Currently no TEEs associated with the use of domestically manufactured IVIG have been reported in China. To study the safety of the IVIG produced by the domestic industry, we investigated the predominant plasma proteases in 42 IVIG lots from 4 domestic manufacturers using one-stage clotting assays, TGT, and non-activated partial thromboplastin time (NAPTT). This study describes a rigorous, systematic investigation of FXIa as an impurity in IVIG from 4 Chinese blood fractionation companies. We expect the results of the study to lead to an increased understanding of the safety of the IVIG and stimulate further studies on quality monitoring of other blood products.

\section{MATERIAL AND METHODS}

\section{IVIG lots}

Forty-two 5\% IVIG lots were collected from Chinese blood fractionation companies. All of the lots were within their labeled shelf life.

\section{Quantitative determination of coagulation factors clotting activity}

FII, FVII, FIX, FX, FXI, and FXII activity assays were determined with one-stage clotting assays (The National Committee for Clinical Laboratory Standards, 1997) performed using a CA-1500 Hemostasis Analyzer (Sysmex, Japan) with coagulation factor-deficient plasma (Sysmex). For each factor, a working reference curve of at least 5 dilutions was pre- 
pared with a calibrator plasma and dilution buffer (all from Chengdu Union, China). Samples (IVIG products) were tested at 3 independent dilutions prepared with dilution buffer. The reference and sample results were inspected for parallelism and linearity. A minimum of one sample test result needed to fall within the readable range of the working reference curve to be an acceptable, quantifiable result and calculated as the average of the 3 dilutions assayed. Results are reported in international units per milliliter.

\section{Quantitative determination of FXIa}

Plasma was obtained from healthy individuals via clear venipuncture and by carefully drawing 9 volumes of blood into 1 volume of $106 \mathrm{mM}$ sodium citrate supplemented with corn trypsin inhibitor (Merck Biosciences, Germany) in a final concentration of 100 $\mu \mathrm{g} / \mathrm{mL}$. After centrifugation for $20 \mathrm{~min}$ at $800 \mathrm{~g}$ at room temperature, the upper half of the supernatant was withdrawn and centrifuged again at $4000 \mathrm{~g}$ for $20 \mathrm{~min}$ at room temperature. The upper half of the supernatant was then collected and frozen in small aliquots at $-80^{\circ} \mathrm{C}$. the TGT was performed in triplicate according to procedures published (Grundmann et al., 2010) and with the following modifications: $6.25 \mu \mathrm{L} \mathrm{5 \%} \mathrm{IVIG} \mathrm{solution} \mathrm{preparation} \mathrm{to} \mathrm{be}$ investigated was added to $25 \mu \mathrm{L}$ plasma obtained as described above. The reaction mixture was made up to $50 \mu \mathrm{L}$ by adding $\mathrm{NaCl}$ HEPES buffer containing $2.5 \mu \mathrm{M}$ cephalin and 10 $\mathrm{mM}$ substrate. After a 5 -min pre-incubation at $37^{\circ} \mathrm{C}$, the reaction was started by adding 50 $\mu \mathrm{L} 25 \mathrm{mM}$ pre-warmed $\mathrm{CaCl}_{2}$ solution in buffer. Thrombin generation was monitored for 1 $\mathrm{h}$ by measuring the developing fluorescence at 390 and $460 \mathrm{~nm}$ (excitation/emission) every $20 \mathrm{~s}$. A blank curve (containing all ingredients plus ethylenediaminetetraacetic acid in excess to calcium) was subtracted from all measurements. A dilution series of FXIa (30 to $0 \mathrm{nM}$ ) served as a calibrator.

\section{Determination of NAPTT}

NAPTTs were performed as described in the European Pharmacopoeia on samples from four manufacturers and tested after dilutions of 1:5 in the assay buffer. Briefly, $30 \mu \mathrm{L}$ human virus-inactivated pooled plasma was mixed with an equal volume of cephalin (Merck Biosciences). After $60 \mathrm{~s}$ at $37^{\circ} \mathrm{C}, 30 \mu \mathrm{L} 1: 10$ and 1:100 sample dilutions in Tris buffer, $\mathrm{pH}$ 7.5, and $30 \mu \mathrm{L} 25 \mathrm{mM} \mathrm{CaCl}_{2}$ were added and the clotting time measured. Assays were performed using a CA-1500 Hemostasis Analyzer (Sysmex). Results are reported as seconds between products and normal plasma control included in the same run of the test according to the European Pharmacopoeia monograph (which must yield clotting times between 200 and $350 \mathrm{~s}$ ).

\section{RESULTS}

\section{Coagulation factor clotting activities}

As shown in Table 1, clotting activities were below the detection limit of the assays for all products and coagulation factors (detection limits: $<0.016$ for FX, FXI, and FXII; $<0.013$ for FII and FVII; $<0.015$ for FIX) except for the products of Manufacturer B (lots of 2010), in which 0.030 to $0.032 \mathrm{IU} / \mathrm{mL}$ FXI:C $(\mathrm{N}=5)$ were detected. 
Table 1. Clotting activities determined on the products studied by one-stage clotting assays.

\begin{tabular}{|c|c|c|c|c|}
\hline Clotting activities & Manufacturer A & Manufacturer B & Manufacturer C & Manufacturer D \\
\hline \multicolumn{5}{|l|}{$\overline{\text { FII }}$} \\
\hline Number & 15 & 17 & 4 & 6 \\
\hline $\mathrm{IU} / \mathrm{mL}$ & * & * & * & * \\
\hline \multicolumn{5}{|l|}{ FVII } \\
\hline Number & 15 & 17 & 4 & 6 \\
\hline $\mathrm{IU} / \mathrm{mL}$ & * & * & * & * \\
\hline \multicolumn{5}{|l|}{ FIX } \\
\hline Number & 15 & 17 & 4 & 6 \\
\hline $\mathrm{IU} / \mathrm{mL}$ & * & * & * & * \\
\hline \multicolumn{5}{|l|}{ FX } \\
\hline Number & 15 & 17 & 4 & 6 \\
\hline $\mathrm{IU} / \mathrm{mL}$ & * & * & * & * \\
\hline \multicolumn{5}{|l|}{ FXI } \\
\hline Number & 15 & 12 & 4 & 6 \\
\hline $\mathrm{IU} / \mathrm{mL}$ & $*$ & * & * & * \\
\hline \multicolumn{5}{|l|}{ FXII } \\
\hline Number & 15 & 17 & 4 & 6 \\
\hline $\mathrm{IU} / \mathrm{mL}$ & * & * & $*$ & * \\
\hline
\end{tabular}

*Denotes measurement below assay detection limit.

\section{TGT determination}

The results of TGT determination are shown in Figure 1. It appears that triggering the reaction with FXIa generates a constant amount of thrombin when a certain threshold of FXIa $(30-0 \mathrm{nM})$ is exceeded (see Figure 1A). In addition, the peak time of thrombin (TTP) varied with FXIa concentration over the entire range tested (see Figure 1B), which demonstrates a clearly negative correlation between FXIa concentration and TTP. Measurements of TTP using the TGT in IVIG lots were above 35 min (Table 2).

A

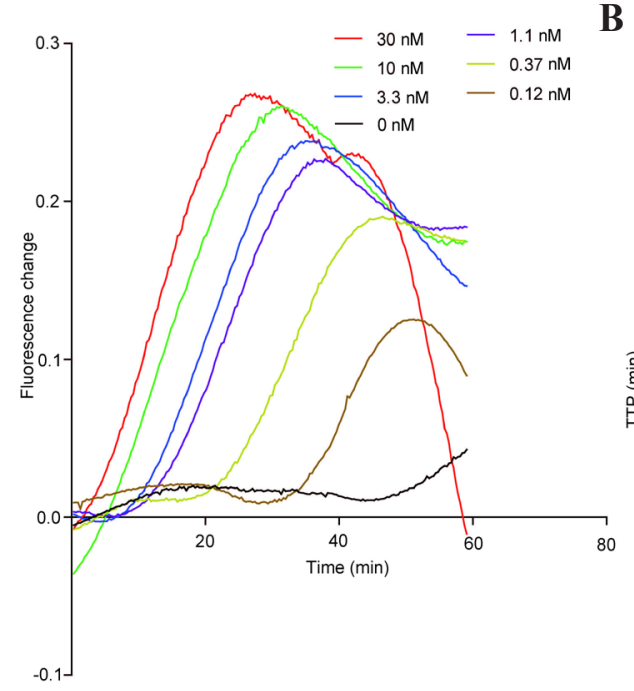

B

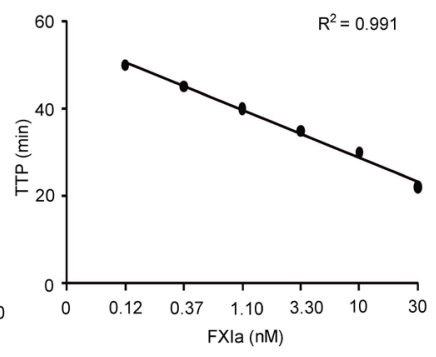

Figure 1. Influence of varying concentrations of residual activated coagulation factor XI (FXIa) on thrombin generation test (TGT). A. TGT was performed with corn trypsin inhibitor-treated plasma. FXIa (30-0 nM) served as trigger. B. All FXIa concentrations tested are plotted against the peak time of thrombin (TTP) in a linearity plot. 
Table 2. Non-activated partial thromboplastin time (NAPTT), peak time of thrombin (TTP), and the concentration of residual activated coagulation factor XI (FXIa) using thrombin generation test performed on intravenous immunoglobulin products.

\begin{tabular}{lcccc}
\hline Product & Number & NAPTT & TTP & FXIa \\
\cline { 2 - 5 } & & Range $(\mathrm{s})$ & Min $($ means \pm SD) & nM (means \pm SD) \\
\hline Manufacturer A & 15 & $204-222$ & $45 \pm 4.0$ & $0.17 \pm 0.08$ \\
Manufacturer B & 17 & $205-223$ & $45 \pm 4.0$ & $0.13 \pm 0.10$ \\
Manufacturer C & 4 & $203-233$ & $42 \pm 5.0$ & $<0.12$ \\
Manufacturer D & 6 & $208-224$ & $37 \pm 2.0$ & $0.19 \pm 0.03$ \\
\hline
\end{tabular}

Data are the means of 3 independent experiments. The concentration of FXIa was calculated relative to a purified FXIa calibration curve. $\mathrm{SD}=$ standard deviation.

\section{NAPTT determination}

Using NAPTT, we determined that the clotting times with four 5\% IVIG lots from 2009 manufactured by the Chinese blood fractionation industry were similar to those of the assay controls, with values ranging from 203 to $233 \mathrm{~s}$ for 1:5 dilution samples (see Table 2).

\section{DISCUSSION}

Thromboembolism is a serious event that harms human health. The study of thromboembolic factors is a focus of blood disease research. Previous studies have shown that functional FXIa, as a major contaminant in IVIG lots involved in TEEs, is first suspected and confirmed and then quantified (Wolberg et al., 2000). Grundmann et al. (2010), Marzo et al. (2011), and Etscheid et al. (2012) have reported that the readout parameter TTP is below 10 min, FXIa concentration is above $0.5 \mathrm{nM}$, FXI clotting activity is detected $(0.08-0.10 \mathrm{IU} / \mathrm{mL})$, and NAPTT is below $150 \mathrm{~s}$ with IVIG batches associated with TEEs, but batches from other manufacturers exhibit TTP values above 30 min, FXIa concentration below $0.5 \mathrm{nM}$, FXI clotting activity below $0.04 \mathrm{IU} / \mathrm{mL}$, and NAPTT above $200 \mathrm{~s}$.

In the current study, we systematically investigated the predominant plasma proteases across 42 IVIG lots from 4 domestic manufacturers. The results showed that the products had less prothrombotic capacity, a result consistent with data from IVIG batches redundant for TEEs (Grundmann et al., 2010; Marzo et al., 2011), but inconsistent with findings of Grundmann et al. (2010), who suggested that lots from specific manufacturers showed a potential prothrombotic profile. A reason for the difference may be due to the production process of IVIG.

For commercial large-scale purification of IVIG, blood fractionation companies modify the manufacturing steps to improve the purity, utilization ratio, and viral safety of the products. However, these modifications introduce the risk of contaminant plasma proteins that are of no concern in the quality and efficacy of the products. Therefore, discussion is still needed to determine whether the current set of quality control tests for immunoglobulins is sufficient to assure a high-quality product that maintains safety and efficacy. We suggest that, at minimum, a global clotting test should be mandatory to assure a low level of prothrombotic activity caused by FXIa and to increase the safety of immunoglobulin products. An NAPTT assay appears suitable to identify prothrombotic potential in IVIGs. In addition, a fluorescence-based modified thrombin generation assay may be a promising alternative to NAPTT, as it is also a global assay and highly sensitive to the FXIa. A modified FXIa assay would be a helpful assay 
to identify specifically elevated levels of the FXIa in IVIG lots.

In summary, our study is the first detailed and comprehensive determination of the FXIa as an impurity in IVIG from Chinese blood fractionation companies. The developed sensitive and modified TGT assays have revealed less prothrombotic capacity than previously reported by the industry; therefore, quantitative determination of FXIa as an impurity in IVIG might be overstated, and it is still unclear whether the FXIa screening of IVIG in China should be required. We will launch a large-scale study to examine additional IVIG samples using TGT in collaboration with Chinese blood fractionation companies.

\section{ACKNOWLEDGMENTS}

Research supported by a grant of the Ministry of Health of China (\#200902008-02).

\section{REFERENCES}

Bolton-Maggs PH, Colvin BT, Satchi BT and Lee CA (1995). Thrombogenic potential of factor XI concentrate. Haemophilia 1: 283-285.

Brannagan TH, III, Nagle KJ, Lange DJ and Rowland LP (1996). Complications of intravenous immune globulin treatment in neurologic disease. Neurology 47: 674-677.

Cochrane CG and Griffin JH (1979). Molecular assembly in the contact phase of the Hageman factor system. Am. J. Med. 67: 657-664

Dalakas MC (1994). High-dose intravenous immunoglobulin and serum viscosity: risk of precipitating thromboembolic events. Neurology 44: 223-226.

Etscheid M, Breitner-Ruddock S, Gross S, Hunfeld A, et al. (2012). Identification of kallikrein and FXIa as impurities in therapeutic immunoglobulins: implications for the safety and control of intravenous blood products. Vox Sang. 102: 40-46.

Grundmann C, Kusch M, Keitel S, Hunfeld A, et al. (2010). Modified thrombin generation assay: application to the analysis of immunoglobulin concentrates. WebmedCentral IMMUNOTHERAPY 11: WMC001116.

Hefer D and Jaloudi M (2004). Thromboembolic events as an emerging adverse effect during high-dose intravenous immunoglobulin therapy in elderly patients: a case report and discussion of the relevant literature. Ann. Hematol. 83: 661-665.

Marzo N, José M, Lopez L, Bono M, et al. (2011). Quantitative determination of relevant amounts of blood coagulation factor XI activity in a specific brand of intravenous immunoglobulin. WebmedCentral IMMUNOTHERAPY 5: WMC001922.

Rosenbaum JT (1997). Myocardial infarction as a complication of immunoglobulin therapy. Arthritis Rheum. 40: 1732-1733.

The European Pharmacopoeia (2009). The Directorate for the Quality of Medicines and Healthcare of the Council of Europe, Strasbourg.

The National Committee for Clinical Laboratory Standards (1997). The Determination of Factor Coagulant Activities. H-48-A.17, Wayne.

Vo AA, Cam V, Toyoda M, Puliyanda DP, et al. (2006). Safety and adverse events profiles of intravenous gammaglobulin products used for immunomodulation: a single-center experience. Clin. J. Am. Soc. Nephrol. 1: 844-852.

Wolberg AS, Kon RH, Monroe DM and Hoffman M (2000). Coagulation factor XI is a contaminant in intravenous immunoglobulin preparations. Am. J. Hematol. 65: 30-34. 